\title{
Dielectrophoretic tweezer for isolating and manipulating target cells.
}
A. Menachery ${ }^{1,2}$, D. Graham ${ }^{2}$, S. M. Messerli², R. Pethig ${ }^{1,2}$, P. J. S. Smith ${ }^{2}$
${ }^{1}$ Institute for Integrated Micro and Nano Systems, Joint Research Institute for Integrated Systems, School of Engineering, The University of Edinburgh, Edinburgh, EH9 3JF, UK
${ }^{2}$ Biocurrents Research Center, Marine Biological Laboratory, Woods Hole, MA 02543, USA

\begin{abstract}
:
The ability to isolate and accurately position single cells in three dimensions is becoming increasingly important in many areas of biological research. We describe the design, theoretical modeling and testing of a novel dielectrophoretic (DEP) tweezer for picking out and relocating single target cells.. The device is constructed using facilities available in most electrophysiology laboratories, without the requirement of sophisticated and expensive microfabrication technology, and offers improved practical features over previously reported DEP tweezer designs. The DEP tweezer has been tested using transfected HEI 193 human schwannoma cells, with visual identification of the target cells being aided by labeling the incorporated gene product with a green fluorescent protein.
\end{abstract}

Communicating author: Ron.Pethig@ed.ac.uk 


\section{Introduction}

The objective of this work was to develop a method to make low-throughput cell picking cheaper, less destructive and more readily available compared to bulk cell sorters such as fluorescent activated cell sorting (FACS). Applications would include the ability to pick fewer or rare cells (such as primary cells, stem cells or fungi and protists) from native environments, and to pick or position particular and fragile cells for experimental purposes. We have chosen to incorporate an electrokinetic effect known as dielectrophoresis (DEP) into the form of an electronic tweezer.

The application of DEP described here provides a novel method for producing stably transfected cell lines through its ability to selectively isolate transfected from nontransfected cells. While traditional methods for production of stably transfected cell lines involve transfection of cells and selection by drug susceptibility, this new procedure has the potential to simplify and shorten the process. We have tested the practical use of the DEP tweezer by demonstrating that it can be used to isolate schwannoma cells expressing a membrane channel protein of interest, tagged to the green fluorescent protein (GFP), from nontransfected cells. The use of a DEP tweezer is not dependent on a visible fluorescent marker but can be used with such markers. It can be modified to accommodate cells of different sizes and shapes, and focus attention to rare cells. The method can also be electronically automated (using a robotic arm and a programmed electronic signal generator) and integrated with imaging to increase throughput for cell picking.

Dielectrophoresis (DEP) is a well-proven technique for moving particles using AC electrical field gradients [1]. The simplest electromanipulation device, utilizing DEP, would consist of a 
single wire. Schnelle et al demonstrated that yeast cells could be attracted to a single gold wire, capacitively coupled to a counter electrode [2]. However, maintaining a consistent DEP force using capacitive coupling to a moveable single wire is difficult, as is the selective trapping of a single cell from a collection of cells. In a variation of this single wire design, Lee et al have recently demonstrated that yeast cells and erythrocytes can be manipulated in three dimensions using an electrode arrangement comprising an electrochemically sharpened tungsten wire positioned above a planar electrode [3]. However, the DEP force generated at the wire tip is very sensitive to its height above the planar electrode, and there is a lack of spatial accuracy for cell capture and release. Also, the fact that a captured cell is held by a single point of contact will limit the extent to which it can resist fluid drag forces, and hence limit the speed at which it can be translocated through a fluid medium.

The first reported design of a twin-electrode DEP tweezer for manipulating cells took the form of a microring-ring electrode assembly formed by the vacuum deposition of platinum and gold films onto the outer and inner surfaces, respectively, of a $6 \sim 10 \mu \mathrm{m}$ diameter capillary tip [4]. Single myeloma cells could be trapped by positive DEP, but this was found to result in cell damage arising from electroporation of the cell membrane [4]. With a later design, in the form of a dual-microdisk comprising two platinum-rhodium electrodes of diameter $\sim 2 \mu \mathrm{m}$, single chlorella cells were captured by positive DEP and released by negative DEP at a new location, without harming the cell [5]. Hunt and Westervelt [6] have also demonstrated that a DEP tweezer, fabricated by vacuum evaporating electrically isolated Ti-Au films onto two sides of a sharpened glass rod, can trap yeast cells for several hours without harming them (the trapped cells were observed to bud and form daughter cells). The DEP tweezer design reported here 
offers advantages in terms of its relatively simple fabrication. We also provide modeling data for maximizing its practical application in selectively manipulating single cells.

\section{Experimental}

\subsection{DEP Tweezer}

Gold wires of diameter $25 \mu \mathrm{m}$ and length $\sim 1.5 \mathrm{~cm}$ were electrochemically etched in $1 \mathrm{M} \mathrm{HCl}$ using $4 \mathrm{~V}$ square wave electrical pulses at $2.5 \mathrm{kHz}$, according to the method described by Woo et al [7]. By varying the immersion depths and the holding time of the wire in the etchant, a roughly hemispherical tip and a gradual taper along the wire could be achieved. The etching process was continuously monitored through a microscope, and the two parameters of interest, namely the tip radius (r) and the taper angle ( $\theta$ ), are shown in figure 1(a). The non-etched end of the gold wire was then bonded to a $0.5 \mathrm{~mm}$ diameter stainless steel syringe needle using silver epoxy resin. The DEP tweezer was formed by inserting a syringe needle plus bonded gold wire along each half of a $\sim 5 \mathrm{~cm}$ length of a theta glass capillary $(1.5 \mathrm{~mm} \mathrm{OD}, 0.23 \mathrm{~mm}$ Wall, 0.17 mm Septum; World Precision Instruments, Sarasota, Florida). As shown in figure 1(a), the internal glass septum of the theta glass capillary acted as an electrical insulator between the two wires. One end of the capillary had previously been tapered using a Flaming/Brown style micropipette puller (Sutter Instrument, Novato, CA) to allow the etched ends of two gold wires to protrude slightly and form the tips of the DEP tweezer. The two gold wire tips shown in figure 1 (b) were etched to have similar tip radii and carefully positioned (to within $\pm 1 \mu \mathrm{m}$ ) to extend the same distance from the capillary tip. The ends of the capillary were then sealed using a UV-sensitive epoxy resin. 


\subsection{Cell Samples}

Immortalized human schwannoma cells, HEI 193 [House Ear Institute, 8] were cultured in Dulbecco's modified Eagle's medium (DMEM, Cellgro, Herndon, VA) containing serum-free N-2 Supplement (Gibco), $2 \mu \mathrm{M}$ forskolin (Calbiochem, San Diego, CA), $14 \mathrm{ng} / \mathrm{ml}$ of recombinant human glial growth factor (rhGGF2, Sigma), $50 \mu \mathrm{g} / \mathrm{ml}$ gentamicin, $10 \%$ fetal bovine serum (FBS, Sigma) and $100 \mu \mathrm{g} / \mathrm{ml}$ penicillin/streptomycin (P/S, Cellgro), and plated onto plastic dishes $(75 \mathrm{~mm})$. All cells were grown at $37^{\circ} \mathrm{C}$ in a $95 \%$ air $/ 5 \% \mathrm{CO}_{2}$ atmosphere.

The cells were transfected with a construct which contained a $\mathrm{Ca} \beta$ subunit cloned into the pXOOM vector. In order to identify cells expressing the incorporated gene product, a construct was used which included a green fluorescent protein (GFP) marker gene $[9,10]$. The cells were transfected with Lipofectamine 2000 (Invitrogen) in cultures grown in $75 \mathrm{~mm}^{2}$ plastic culture flasks (Fisher). The transfected cell lines exhibited different levels of expression depending on the quantity of the plasmid introduced into the cell.

\subsection{Dielectrophoresis (DEP) Measurements}

To test the DEP tweezer using HEI-193 schwannoma cells, knowledge of the DEP behavior of these cells was required. It was necessary to predict, for set suspending solution conductivities, the voltage frequencies required to first attract a cell to the tweezer by a positive DEP force and to then release and repel the cell by negative DEP. This information was obtained using a procedure described previously [11]. In brief, cells at a working concentration of $\sim 10^{5}$ cells $/ \mathrm{mL}$, were pipetted onto a quadrupole-electrode assembly fabricated onto a microscope slide, and 
secured with a cover slip. To reduce the adherence of cells to the glass slide, the surface was passivated with a polyethylene glycol coating (MicroSurfaces Inc). Digitally generated voltages of frequencies between $10 \mathrm{kHz}$ and $10 \mathrm{MHz}$, produced using a custom-built generator, were applied to the electrodes. The applied voltage frequency was adjusted to cause the cell under examination to sequentially make transitions between positive and negative DEP, and these responses were visualized using a Zeiss Axioskop and recorded for later analysis of these transitions. The measurements were performed at $37^{\circ} \mathrm{C}$ ). The objective was to determine the DEP cross-over frequency for a representative number of cells $(n \approx 30)$ for a range of cell suspension conductivities. The DEP cross-over frequency marks the transition where the DEP force acting on a cell changes from negative to positive DEP, and is a sensitive function of the physico-chemical properties of a cell and its environment [12]. It was determined either by finding the frequency where a cell became stationary or by interpolating the estimated cell velocities to find the frequency where the DEP force was zero. An integrated micrometer scale was used to determine cell diameter to an accuracy of $\pm 0.5 \mu \mathrm{m}$.

Immediately prior to the DEP experiments, the adherent HEI-193 cells were detached from the bottom of their culture flask using trypsin, then re-suspended and washed twice in the DEP test solution. This trypsinization procedure, required because DEP measurements cannot be made on adherent cells, is not known to cause significant changes in the dielectric properties of a cell. DEP test solutions were prepared to different conductivities in the range 19 to $115 \mathrm{mS} / \mathrm{m}$ by addition of phosphate buffered saline solution to de-ionized water. The solution was then adjusted to $\mathrm{pH} 7.4$ by adding $\mathrm{NaOH}$ and the osmolarity adjusted to physiological levels of around $300 \mathrm{mOsm} / \mathrm{kg}$ by adding sucrose. The osmolarity was measured using an osmometer 
(Vapro 5520, Wescor Inc., Utah) calibrated using Opti-Mole standard $\mathrm{NaCl}$ solutions, and the final conductivities were measured using a YSI 3200 (Yellow Springs, Ohio) conductivity meter.

\subsection{DEP Tweezer Tests}

The tests were performed using an inverted fluorescence microscope (Zeiss Axioscope) equipped with a micromanipulator (Narishiga). The microscope was housed within a chamber heated to $37^{\circ} \mathrm{C}$ to minimize thermal shock to the cells, and the experiments were monitored and recorded using a video camera. The experimental arrangement is shown in figure 2. $200 \mu$ of the DEP solution was pipetted onto a substrate coated with a $\sim 8 \mathrm{~nm}$ thick layer of polyethyleneglycol. The tip of the DEP tweezer, located in the micromanipulator at an angle of $45^{\circ}$ to the horizontal, was lowered into this solution. $10 \mu \mathrm{l}$ of the HEI-193 cell suspension, at a density of $1 \mathrm{x}$ $10^{6} \mathrm{cell} / \mathrm{s} / \mathrm{ml}$, was carefully pipetted onto the substrate such that the spread of the cells upon settling onto the substrate was minimal. Target cells were located by scanning the substrate and identifying those exhibiting a relatively high fluorescence intensity. The tip of the DEP tweezer was brought to within 15 20 microns of a target cell, then electrically energized to attract and trap it by positive DEP between the two gold wire tips. By careful positioning of the tweezer tip a target cell, either in close proximity to or in contact with another cell, could be attracted and trapped. The trapped cell was then moved to a new location $\sim 5 \mathrm{~mm}$ away from the other cells, and released by applying a $10 \mathrm{kHz}$ signal to produce a negative DEP force. This new location could either be a region on the substrate from where the target cell could be extracted using a micropipette, or (as shown in figure 2) within a microelectrode array for further DEP or electrorotation characterisation, for example. 


\section{Results and Discussion}

\subsection{DEP Measurements}

The range of DEP cross-over frequencies observed for the HEI-193 cells, as a function of suspending medium conductivity, is shown in figure 3 . Theory $[11,12]$ predicts the observed linear relationship shown in figure 3 , with the slope of the straight line given by:

$$
\text { Slope }=\frac{\sqrt{2}}{2 \pi R C_{m}}
$$

where $R$ is the mean cell radius and $C_{m}$ is the cell membrane capacitance. The slope of the plot shown in figure 3 is $1.52 \mathrm{~m} / \mathrm{F}$, and a value of $6 \pm 1.5 \mu \mathrm{m}$ was determined for the mean radius ( $\mathrm{n}$ $=120$ ) of the HEI-193 cells. From equation (1) this gives a value of $24.7 \pm 6.2 \mathrm{mF} / \mathrm{m}^{2}$ for $C_{m}$.

Observed values for the plasma membrane capacitance of various cell types range from $\sim 8$ to $>40 \mathrm{mF} / \mathrm{m}^{2}$, and correlate closely with the extent to which the area of an otherwise smooth membrane surface is increased as a result of membrane folding and protuberances [13].

Based on this derived value of $24.7 \mathrm{mF} / \mathrm{m}^{2}$ for the membrane capacitance of the HEI-193 cells, their DEP response can be predicted as a function of voltage frequency and the conductivity of the cell suspending solution. In figure 4 the DEP characteristics expected for HEI-193 cells are shown for the lower $(19 \mathrm{mS} / \mathrm{m})$ and upper $(115 \mathrm{mS} / \mathrm{m})$ solution conductivity values used in our tests of the DEP tweezer. The relative DEP response is given as the Clausius-Mossotti polarization factor for the multi-shell model of a cell [11-13], and was modeled using MATLAB 7.4 (The MathWorks, Inc.). Based on figure 4 it was concluded that a voltage frequency of 1 10 MHz should be selected when using the DEP tweezer to attract a cell by positive DEP, and that 
this attracting force is reduced by $\sim 30 \%$ as the solution conductivity is increased from 19 to 120 $\mathrm{mS} / \mathrm{m}$. To release the cell by negative DEP, a frequency of $1 \sim 10 \mathrm{kHz}$ can be used, and the DEP force is relatively insensitive to the solution conductivity. The effect of increasing the solution conductivity to $400 \mathrm{mS} / \mathrm{m}$ is also shown in figure 4 . Whereas the negative DEP force at $1 \sim 10$ $\mathrm{kHz}$ remains relatively unchanged, at $10 \mathrm{MHz}$ there is a $73 \%$ reduction in the DEP force compared to that generated in a $19 \mathrm{mS} / \mathrm{m}$ solution. Modeling such as that shown in figure 4 can be used as an aid to control the positive DEP attracting force in terms of the choice of the signal frequency. It is also evident that operation of the DEP tweezer is limited to solution conductivities no larger than around $0.4 \mathrm{~S} / \mathrm{m}$, which is approximately one-third of that typically exhibited by physiological strength buffers.

\subsection{Cell Manipulation using the DEP Tweezer}

The sequence of images shown in figures 5(a \& b) show the capture of a fluorescent HEI-193 cell in a $19 \mathrm{mS} / \mathrm{m}$ solution. The tip of the DEP tweezer was directed at an angle of $45^{\circ}$ next to a target cell, at a distance of around two cell diameters (i.e., $\sim 20 \mu \mathrm{m}$ ), and then lowered to a height of $10 \sim 15 \mu \mathrm{m}$ above the substrate. A 1 Volt peak, $1 \mathrm{MHz}$, signal was then applied to create an attractive DEP force at the tweezer tip. On initiating cell capture, and after the cell had moved $\sim 7 \mu \mathrm{m}$ from its initial resting position, the signal voltage was lowered to $0.5 \mathrm{~V}$ peak to reduce the electrical stress on the cell. By determining the time taken for the cell to travel the first $2 \mu \mathrm{m}$ after initiation of motion, the initial cell velocity was found to be $1 \sim 2 \mu \mathrm{m} / \mathrm{s}$. By adjusting the micromanipulator, the trapped cell was moved over a distance of $\sim 5 \mathrm{~mm}$ at an average speed of $\sim 100 \mu \mathrm{m} / \mathrm{s}$ without causing it to escape from the tweezers as a result of hydrodynamic drag forces. On locating the tweezer tip at the desired new location in the culture dish, the signal 
frequency was changed to $10 \mathrm{kHz}$ so as to release the trapped cell through the action of negative DEP (figures 5(c \& d). The action of a repulsive, negative, DEP force was required to release a captured cell, and could also be used to reposition a released cell on the substrate.

Tests were performed for various values of the radius of the etched gold wire tip. It was found that there was an inverse relationship between tip radius and the voltage required to initiate cell capture. It was also found that on reducing the tip radius to $\sim 0.5 \mu \mathrm{m}$, the chance of causing cell damage was increased. To understand these observations, theoretical modeling of the DEP force was performed as a function of the tweezer's design parameters.

\subsection{Modeling of DEP Tweezer Performance}

The action of the DEP tweezer depends on the ability to generate a controllable electric field gradient at its tip. Cells located in a region of field non-uniformity will experience a DEP force of magnitude proportional to the product of the local field $E$ and the field gradient $\nabla E$. The imposed electric field induces a surface charge distribution on the cell which, to a good approximation, takes the form of an electric dipole moment whose magnitude and polarity depends on the frequency-dependent dielectric polarizabilities of the cell and surrounding medium. If the field is non-uniform (i.e., there is a local field gradient) a DEP force proportional to the vector parameter $\nabla E^{2}$ will act on this induced dipole moment, causing a freely suspended or non-adherent cell to move [1]. For a fixed value of the applied voltage, the magnitude of $v E^{2}$ is determined mainly by the radius of curvature $r$ of the etched gold wire tips, the spacing $d$ between electrode tips, and the distance $A B$ between an electrode tip and the cell, as shown in figure 1. We have found, within the practical fabrication limits, that the angle of taper $\theta$ and the 
length of gold wire electrode that protrudes from the end of the glass capillary, do not have a significant influence in optimizing the performance of the DEP tweezer.

Modeling of the electric parameter $\nabla E^{2}$ generated by the DEP tweezer design was performed using COMSOL Multiphysics (version 3.5) software. A 3D geometry model of the two etched gold wires was created, with each wire defined as a cone of taper angle $\theta$ having an apex geometry represented as a hemisphere of radius $r$, and with $d$ as the spacing between the wire electrode pairs, as shown in figure 1(a). The 3D model was constructed such that the two wire electrodes were located in a cube of edges $100 \mu \mathrm{m}$ in length, and conventional boundary conditions for the solution of partial differential equations were employed. Neumann boundary conditions (normal component of the electric displacement set to zero) were set on the walls of the cube, and Dirichlet boundary conditions (specifying the electric potentials) were imposed on the two wire electrode surfaces. For the modeling results presented here, potentials of zero and $1 \mathrm{~V}$ (peak) were set for the first and second electrode surfaces, respectively.

A contour plot of $\nabla E^{2}$ is given in figure 6 for a $2 \mathrm{D}$ cross-section through the centers of the wire electrodes, each of tip radius $1 \mu \mathrm{m}$ and with the electrode separation $d$ (as defined in figure 1) set at $4 \mu \mathrm{m}$. As expected, the $\nabla E^{2}$ contours are symmetrical with respect to the central axis between the two electrodes, with a peak value of $\sim 10^{18} \mathrm{~V}^{2} / \mathrm{m}^{3}$ at their tips and reducing rapidly to values of less than $10^{11} \mathrm{~V}^{2} / \mathrm{m}^{3}$ for distances greater than $20 \mu \mathrm{m}$ from the electrodes. It should be noted that, with all other parameters fixed, the value of $\nabla E^{2}$ is directly proportional to the square of the applied voltage. . Thus, lowering the applied voltage from $2 \mathrm{~V}$ to $0.5 \mathrm{~V}$ after cell capture results in a 16-fold reduction of the electrical stress acting on a cell. The influences on the magnitude 
and spatial variation of $V E^{2}$ on changing the radius of curvature $r$ of the gold wire tip, and the electrode separation distance $d$, are shown in figures 7 and 8 , respectively.

To place the modeling results of figures 6-8 into context, we should consider the forces initially involved in attracting a cell, from rest on the substrate, to the DEP tweezer tip. We will assume that the initial induced velocity of $\sim 1 \mu \mathrm{m} / \mathrm{s}$ is clearly greater than any randomizing effects resulting from Brownian motion, and that the dominant forces acting initially on the cell are the DEP force, $F_{D E P}$, and the fluidic viscous drag force, $F_{d r a g}$. From osmotic considerations $[1$, pp.86, 87] the absolute value of maximum diffusional force acting on a particle is $\mathrm{kT} / 2 R$, which for a $6 \mu \mathrm{m}$ radius particle corresponds to a randomizing force of $3.6 \times 10^{-16} \mathrm{~N}$ at $37^{\circ} \mathrm{C}$. We will also assume negligible cell-substrate interactions such as adhesion, and ignore the gravitational buoyancy force because initially the cell experiences more of a lateral than vertical displacement. To estimate the DEP force, the assumption is also made that, at the location of initial cell movement, the length scale of the nonuniformity of the field imposed by the tweezer is large compared to the cell size. This permits use of the standard dipole moment approximation for the DEP force:

$$
F_{D E P}=\pi \varepsilon_{o} \varepsilon_{S} R^{3} C M\left(\nabla E^{2}\right)
$$

where $\varepsilon_{0} \varepsilon_{\mathrm{s}}$ is the absolute permittivity of the solution, $R$ the cell radius, $E$ is the peak amplitude of the electric field, and $C M$ is the real part of the Clausius-Mossotti factor (plotted as a function of frequency in figure 4) describing the effective polarizability of the cell [1]. A particle as small as a cell, subjected to a DEP force $F_{\text {DEP }}$, will almost instantaneously attain a terminal velocity u 
given by [14]

$$
u=F_{D E P} /(6 \pi \eta R)
$$

where $\eta$ is the absolute dynamic viscosity of the solution. From equations $(2 \& 3)$, taking $\varepsilon_{0} \varepsilon_{\mathrm{s}}=$ $7 \times 10^{-10} \mathrm{~F} / \mathrm{m}, C M=0.92$ (from figure 4), $\eta=0.68 \mathrm{mPa} . \mathrm{s}$ (interpolation for water at $37^{\circ} \mathrm{C}$ from ref. 15), and a solution conductivity of $19 \mathrm{mS} / \mathrm{m}$, we can calculate that to capture a cell of radius $6 \mu \mathrm{m}$ and with an initial velocity $\sim 1.5 \mu \mathrm{m} / \mathrm{s}$ requires a value for $v E^{2}$ of $\sim 3 \times 10^{11} \mathrm{~V}^{2} / \mathrm{m}^{3}$. This agrees well with the value shown in figure 8 for a cell situated $\sim 20 \mu \mathrm{m}$ from the tweezer tip, and can be taken as a useful design parameter.

The results of figure 7 suggest an understanding of why the use of a tip radius of $0.5 \mu \mathrm{m}$ and less resulted in relatively uncontrolled cell capture and occasionally to cell destruction. To achieve an imposed $\nabla E^{2}$ value of $\sim 10^{11} \mathrm{~V}^{2} / \mathrm{m}^{3}$ with a tip radius of $0.5 \mu \mathrm{m}$, the tweezer has to be positioned nearer to a cell than for a tip radius of $2 \mu \mathrm{m}$. The rate of increase and final values for $\nabla E^{2}$ attained as the cell approaches the tip are also very much larger, leading to much greater electrical stress on the cell. Increased electrical stress will also arise from the fact that equation (2) will give an underestimate of the DEP force. As a cell approaches the tweezer tip, the length scale of field nonuniformity will approach or exceed the cell size. Multipolar (e.g., quadrupole) contributions will add to the dipolar approximation for the DEP force - but will not alter its frequency dependence [16]. The results of figure 8 indicate that the choice of electrode separation $d$ is not as an important design consideration as the choice of tip radius $r$

\section{Conclusions}


We report the design of a new form of DEP tweezer, constructed using facilities commonly available in electrophysiology laboratories, designed to pick out and relocate single target cells from a cell culture. The tweezer takes the form of two electrochemically etched gold wires, the non-etched ends of which are bonded to stainless steel syringe needles. These were sealed into each half of a tapered theta glass capillary, with the etched ends of the gold wires protruding slightly to form the working tips of the DEP tweezer. It was found that the angle of capillary taper and the length of protrusion of the gold wire electrodes could be altered without significantly changing the performance of the tweezer. For a fixed value of an applied voltage to the gold wire electrodes, the magnitude of an attractive (positive) DEP force exerted on a target cell is determined mainly by the radius of curvature of the etched gold wire tips, the spacing between electrode tips, and their distance from the cell. To avoid unwanted cell damage, the applied voltage should not exceed $2 \mathrm{~V}$ (peak), and preferably be reduced to $\sim 0.5 \mathrm{~V}$ (peak) after cell capture. It was also concluded that a tip radius of less than $0.5 \mu \mathrm{m}$ should not be used. Other guidelines to avoid cell damage from DEP forces indicate that a frequency window, close to the DEP cross-over frequency, should be avoided [17]. Alternatively, a DEP tweezer could be used to selectively destroy target cells by operating in this frequency window and at a high applied voltage.

The tweezer has been tested using transfected HEI 193 human schwannoma cells, with visual identification of the target cells being achieved by labeling an incorporated gene product with a green fluorescent protein. However, the use of a DEP tweezer is not always dependent on the use of a visible fluorescent marker. For example, a non-viable cell with an impaired cytoplasmic membrane does not exhibit negative DEP below $\sim 1 \mathrm{MHz}[12,13,15]$. Non-viable cells can 
therefore be selectively trapped by positive DEP using a tweezer energized at $10 \mathrm{kHz}$ (where viable cells would exhibit negative DEP). The DEP cross-over frequency, where a transition between negative and positive DEP occurs for viable cells, is a sensitive function of a cell's surface morphology and hence its membrane capacitance value[11-13]. By careful tuning of the applied signal frequency, a DEP tweezer could be used to identify and selectively remove those cells whose membrane capacitance is significantly greater than the other cells in a culture. Flanagan et al [18] studied the DEP properties of neural stem cell populations and concluded that the ultimate fate of cells after differentiation can be predicted by distinct changes in their DEP properties before the presence of specific cell-surface proteins (antigens) can be detected. This opens up potentially important applications of DEP, and thus of DEP tweezers also, in stem cell research and therapy [19].

Finally, we find that viable cells can be attracted and trapped by the tweezer, under the influence of a positive DEP force, using an applied voltage frequency of 1 10 MHz. However, this force of attraction is reduced by $\sim 30 \%$ as the solution conductivity is increased from 19 to $120 \mathrm{mS} / \mathrm{m}$, and operation of the DEP tweezer to pick and trap cells is considered to be limited to solution conductivities no larger than around $0.4 \mathrm{~S} / \mathrm{m}$ (i.e., around one-third of that typically exhibited by physiological strength buffers). The captured cell can then be manipulated to a new location, and released under the action of a negative DEP force by reducing the signal frequency to $1 \sim 10$ kHz. The magnitude of this releasing DEP force is relatively insensitive to the solution conductivity.

\section{Acknowledgements:}


We thank Robert Greenberg for the pXOOM vector containing the Caß subunit, Mark Messerli for valuable discussions, and Tamara Clark for the production of figure 2. This work was funded by the NIH: NCRR as a national research resource [P41 RR001395], and by the Alix and Denis Robinson Fund. 


\section{References:}

1. Pohl H.A.: 'Dielectrophoresis', Cambridge University Press: Cambridge, 1978.

2. Schnelle T., Müller T., Hagedorn R., Voigt A., Fuhr G.: 'Single micro electrode dielectrophoretic tweezers for manipulation of suspended cells and particles', Biochim. Biophys. Acta, 1999, 1428, pp. 99-105

3. Lee K., Kwon S.G., Kim S.H., Kwak Y.K.: 'Dielectrophoretic tweezers using sharp probe electrode', Sensors \& Actuators A, 2007, 136, pp. 154-160

4. Matsue T., Matsumoto N., Koike S., Uchida I.: 'Microring-ring electrode for manipulation of a single cell', Biochim. Biophys. Acta, 1993, 1157, pp. 332-335

5. Ogata S., Yasukawa T., Matsue T.: 'Dielectrophoretic manipulation of a single chlorella cell with dual-microdisk electrode', Bioelectrochem., 2001, 54, pp. 33-37

6. Hunt T.P., Westervelt R.M.: 'Dielectrophoresis tweezers for single cell manipulation', Biomed. Microdevices, 2006, 8, pp. 227-230

7. Woo H.D., Kang H., Park M.S.:'Fabrication of nanoscale gold disk electrodes using ultrashort pulse etching', Anal Chem., 2003, 75, pp. 6732-6736

8. Hung, G., Li, X., Faudoa, R., Xeu, Z.,Kluwe, L., Rhim, J. S., Slattery, W., \& Lim, D. (2002). Establishment and characterization of a schwannoma cell line from a patient with neurofibromatosis 2. Int J Oncol 20, 475-482.

9. Jespersen T., Grunnet M., Angelo K., Klaerke D.A., Olesen S.P.: 'Dual-Function Vector for Protein Expression in Both Mammalian Cells and Xenopus laevis Oocytes', BioTechniques, 2002, 32, pp. 536-540 
10. Salvador-Recatalà, V., Schneider T., Greenberg, M. R.: ‘A typical properties of a conventional calcium channel $\beta$ subunit from the platyhelminth Schistosoma mansoni', $B M C$ Physiology, 2008, 8, pp. 1-11

11. Pethig, R., Jakubek, L., Sanger, R.H., Heart, E., Corson, E., Smith, P.J.S.: 'Electrokinetic measurements of membrane capacitance and conductance for pancreatic $\beta$-cells', IEE. Proc.Nanobiotechnol., 2005, 152, pp. 189-193

12. Pethig R., Talary, M.S.: 'Dielectrophoretic detection of membrane morphology changes in Jurkat T-cells undergoing etoposide-induced apoptosis', IET Nanobiotechnol. 2007; 1, pp. 2-9

13. Pethig R.: 'Dielectrophoresis of Biological Cells'. In: Encyclopedia of Surface and Colloidal Science, Taylor \& Francis, New York, 2006; pp. 1719-1736

14. Huang Y., Holzel R., Pethig R., Wang X-B.: 'Differences in the AC electrodynamics of viable and non-viable yeast cells determined through combined dielectrophoresis and electrorotation studies', Phys. Med. Biol., 1992; 37(7), pp. 1499-1517

15. Dean J.A.: 'Lange's Handbook of Chemistry', McGraw-Hill, 15 ${ }^{\text {th }}$ ed., 1999; p. 5.134

16. Jones T B., Washizu M.: 'Multipolar dielectrophoretic and electrorotation theory', J. Electrostatics. 1996; 37, pp.121-134

17. Menachery, A., Pethig, R., 'Controlling cell destruction using dielectrophoretic forces', IEE Proc. Nanobiotechnol., 2005; 152(4), pp. 145-149.

18. Flanagan L A., Lu J., Wang L., Marchenko S A., et al.: 'Unique Dielectric Properties Distinguish Stem Cells and Their Differentiated Progeny', Stem Cells. 2008; 26(3), pp. 656 -65. 19. Pethig R., Menachery A., Pells, S., De Sousa, P.: Dielectrophoresis: 'A review of applications for stem cell research', J. Biomed. Biotechnol., 2010; 182581 (7 pages). 
Figure Legends

Figure 1: (a) Schematic of the DEP tweezer tip to show taper angle $\theta$, radius of curvature $r$ of each tip, the defined distance $d$ between tips, and the fact that the septum of the theta glass capillary electrically separates the two etched gold electrodes. The distance AB is used to model the values for the DEP force parameter of $\nabla E^{2}$ of equation 2. (b) A microscopic image of a DEP tweezer tip. The distance between the two gold wire tips in this image is $\sim 4 \mu \mathrm{m}$.

Figure 2: The experimental arrangement for testing the DEP tweezer consisted of an inverted fluorescence microscope equipped with a micromanipulator and video camera. A voltage function generator was used to energize the DEP electrodes, and in this schematic target cells are shown being selectively picked and then placed within an electrode array for electrokinetic studies.

Figure 3: Variation of the DEP cross-over frequency measured for HEI-193 cells as a function of the conductivity of the suspending solution $(n \approx 20$ for each data point).

Figure 4: The theoretical DEP-frequency characteristics, in terms of the Clausius-Mossotti polarization factor $C M$ of equation 2, are shown for a $6 \mu \mathrm{m}$ diameter HEI-193 cell as a function of solution of conductivity. MATLAB software was used to model these results, and was based on the membrane capacitance value of $24.7 \mathrm{mF} / \mathrm{m}^{2}$ derived from figure 3 .

Figure 5: Images of the manipulation of a fluorescent HEI-193 cell using a DEP tweezer. The etched wire tips were of radius $r \approx 1 \mu \mathrm{m}$, with an electrode separation $\mathrm{d} \approx 4 \mu \mathrm{m}$. The solution conductivity was $19 \mathrm{mS} / \mathrm{m}$. (a) The tweezer tip is positioned $\sim 20 \mu \mathrm{m}$ away from the target cell (radius $\approx 6 \mu \mathrm{m}$ ). (b) The electrodes are energized with a $1 \mathrm{~V}(\mathrm{pk}), 1 \mathrm{MHz}$, to attract the cell by positive DEP. (c) The trapped cell is repositioned to the center of a quadrupole-electrode system. (d) The cell is released by negative DEP on changing the signal frequency to $10 \mathrm{kHz}$. 
Figure 6: A contour plot of the values of $V E^{2}$ generated at the tip of a DEP tweezer, for an applied voltage of $1 \mathrm{~V}$ (peak), tip radius $r$ of $1 \mu \mathrm{m}$ and electrode separation $d$ of $4 \mu \mathrm{m}$.

Figure 7: Plot of the variation of $v E^{2}$ along $\mathrm{AB}$ (as defined in figure 1) as a function of tip radius $r$, for an applied voltage of $1 \mathrm{~V}$ (peak) and electrode spacing $d$ of $4 \mu \mathrm{m}$.

Figure 8: Plot of the variation of $\nabla E^{2}$ along $\mathrm{AB}$ (as defined in figure 1) for electrode separations $d$ of $4 \mu \mathrm{m}$ and $10 \mu \mathrm{m}$, with a fixed applied voltage of $1 \mathrm{~V}$ (peak) and tip radius $r$ of $1 \mu \mathrm{m}$. 
Figure 1
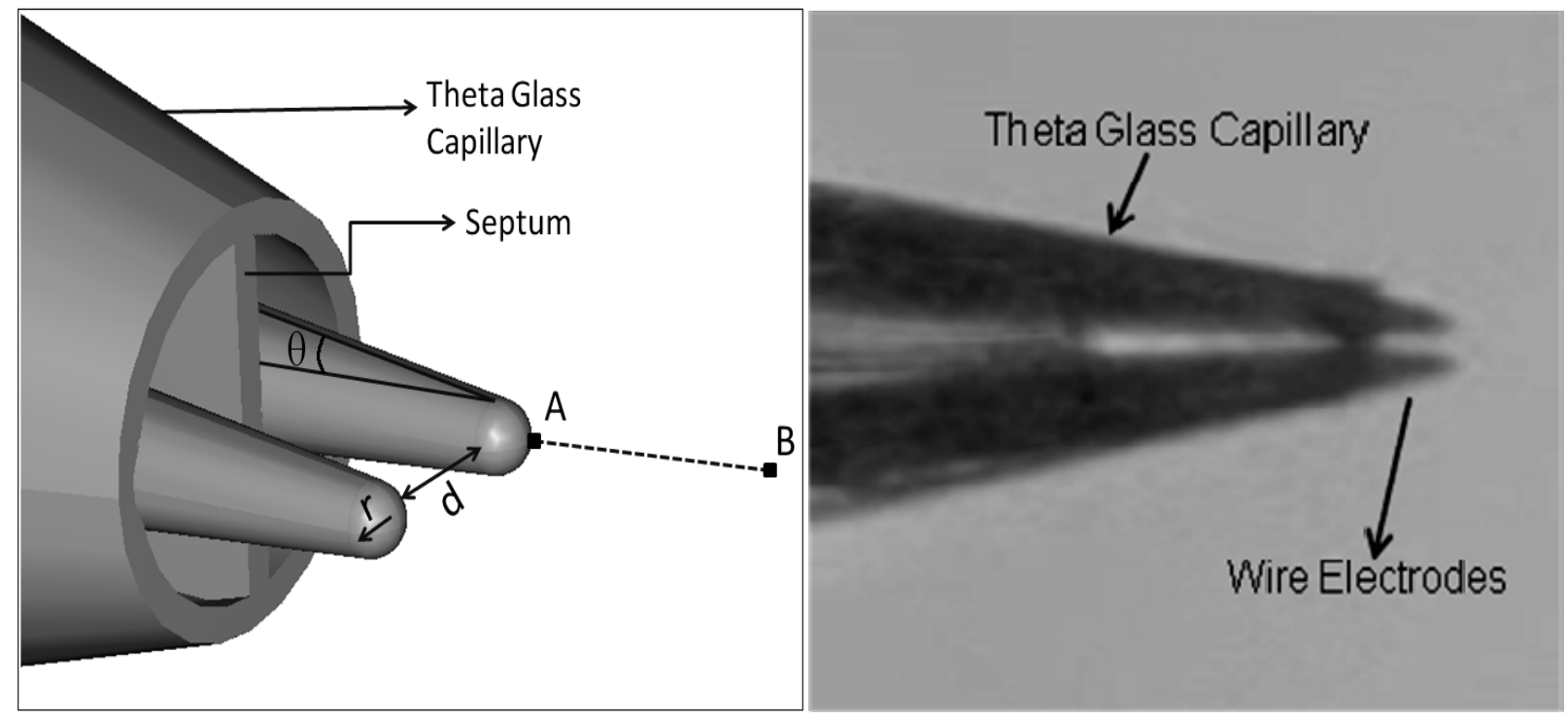

(a)

(b) 
Figure 2

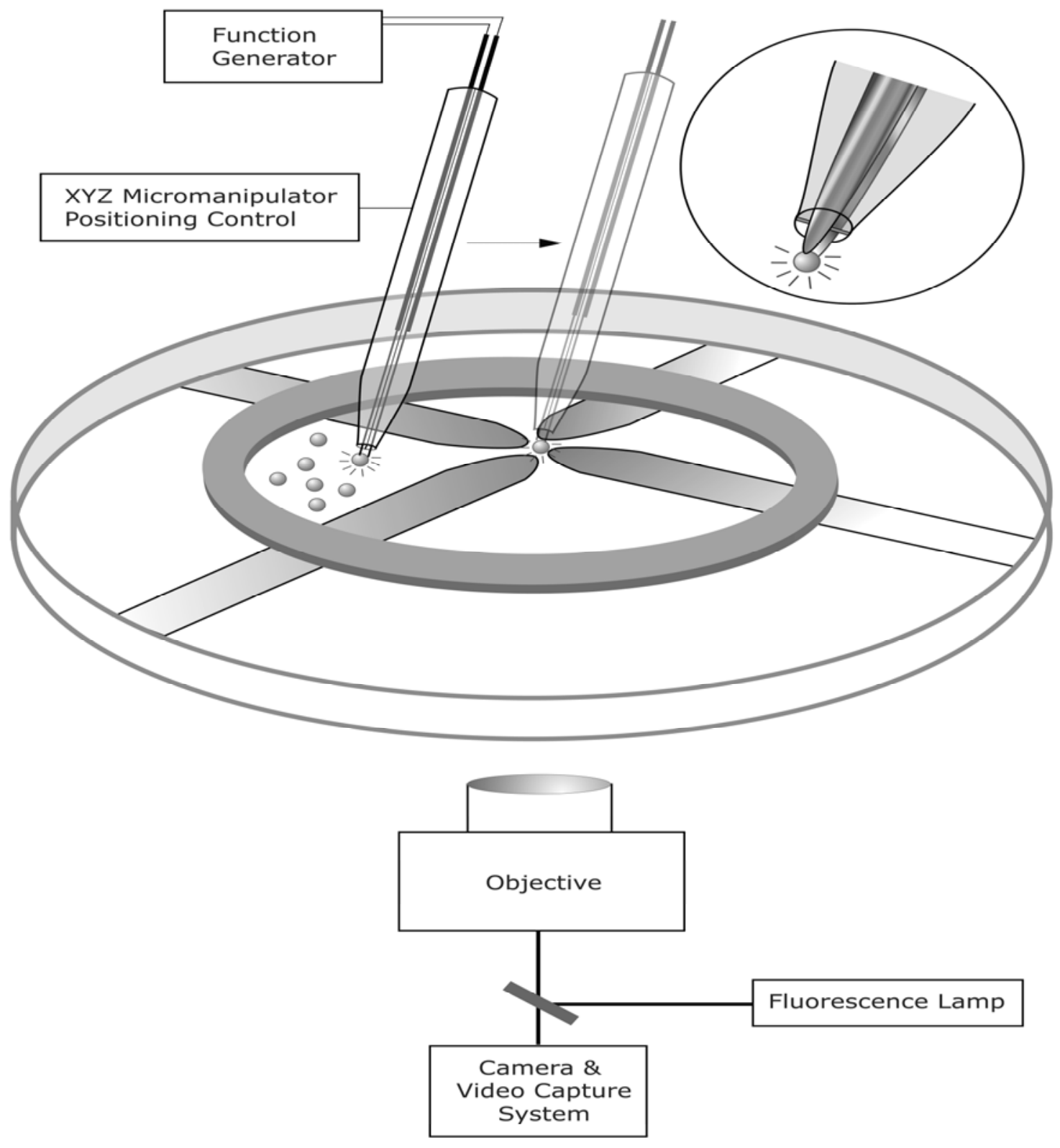


Menachery et al, Dielectrophoretic tweezer

Figure 3

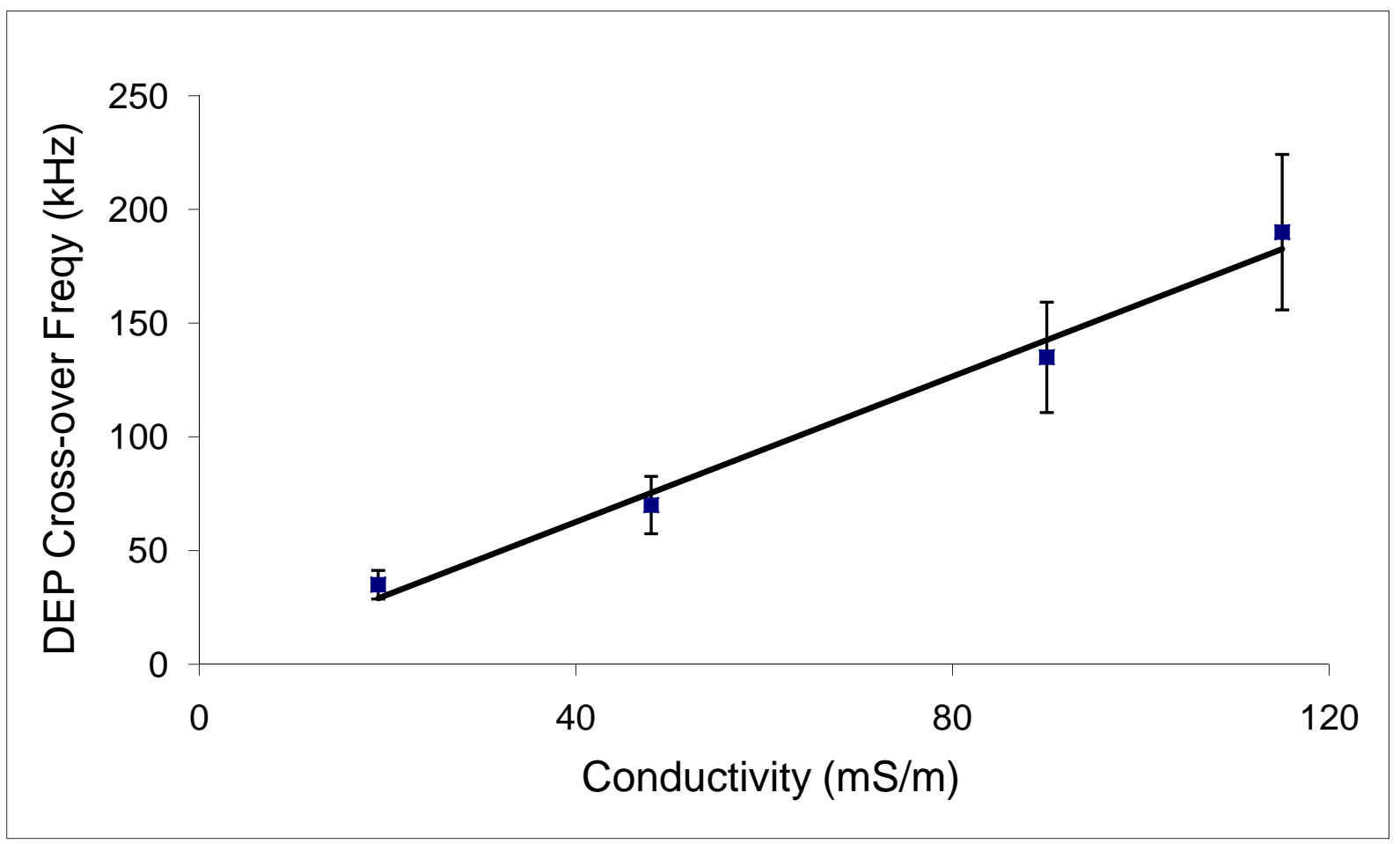


Figure 4

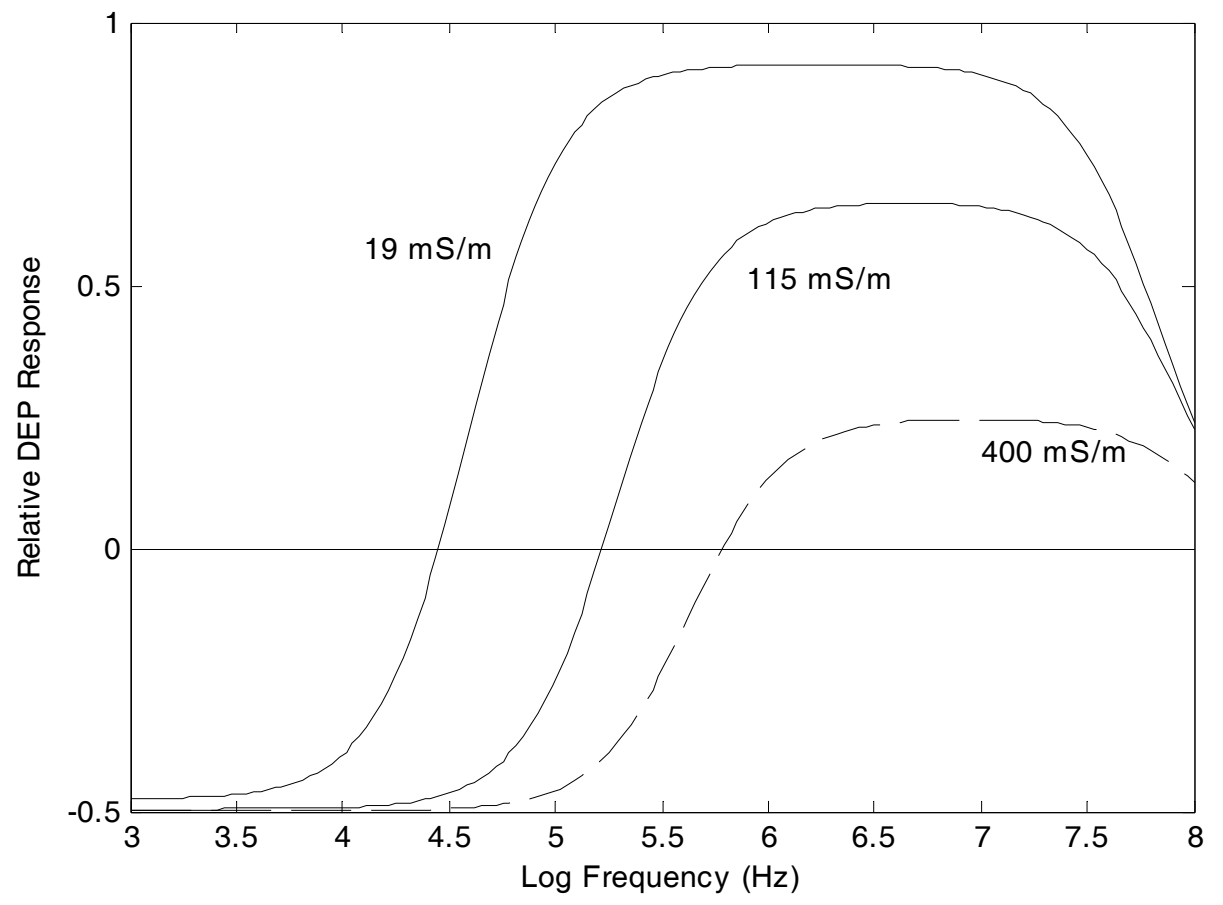


Figure 5

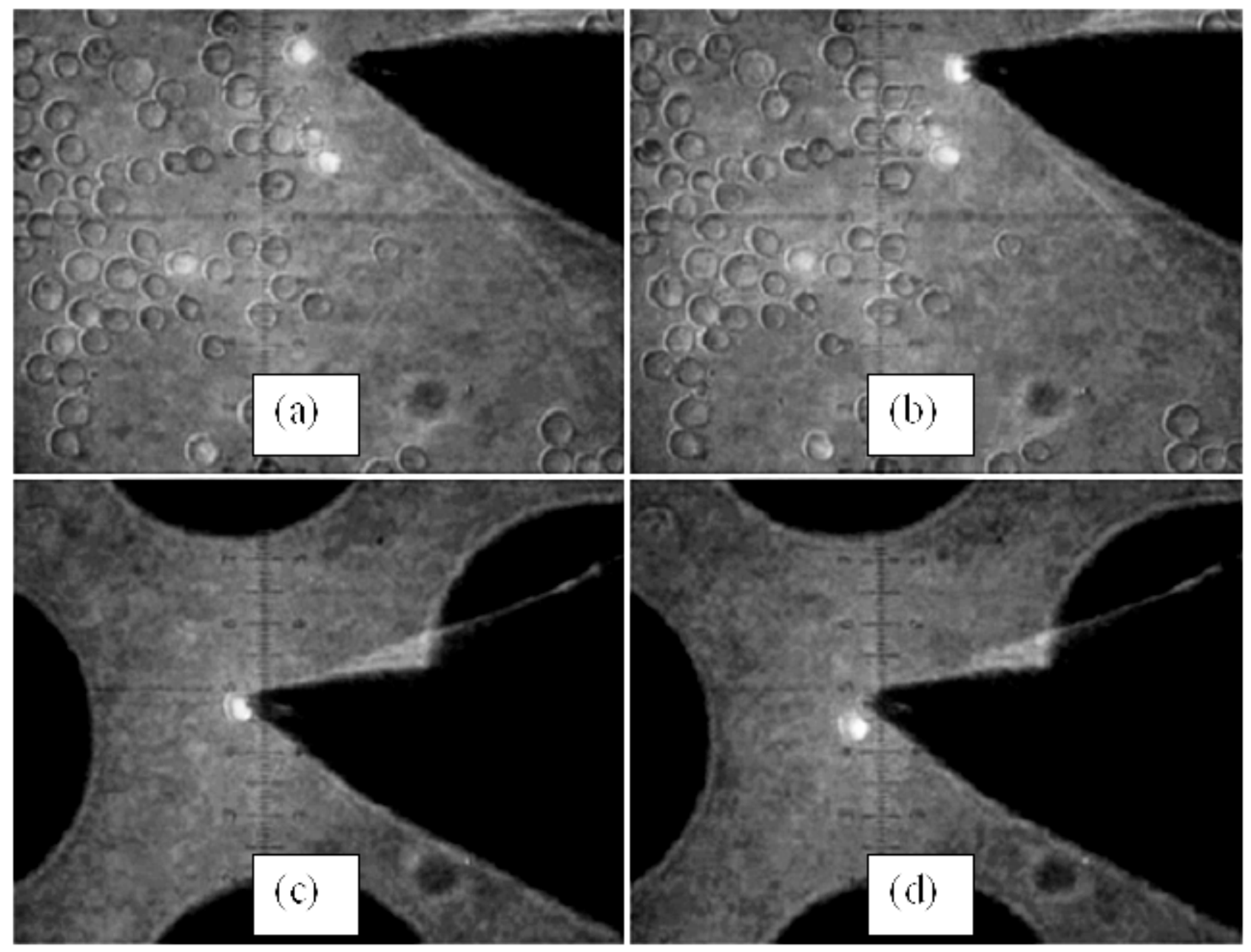


Figure 6

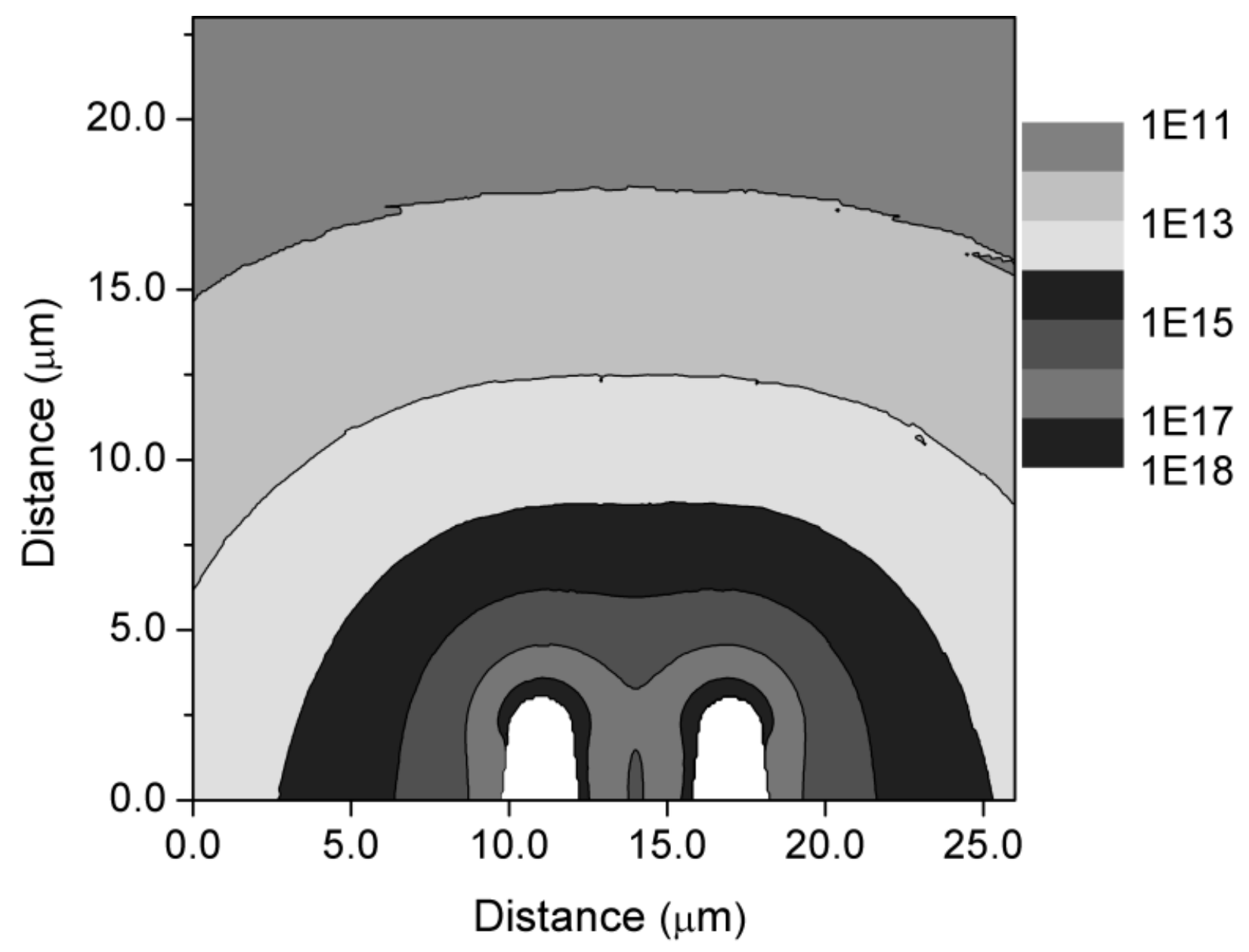


Figure 7

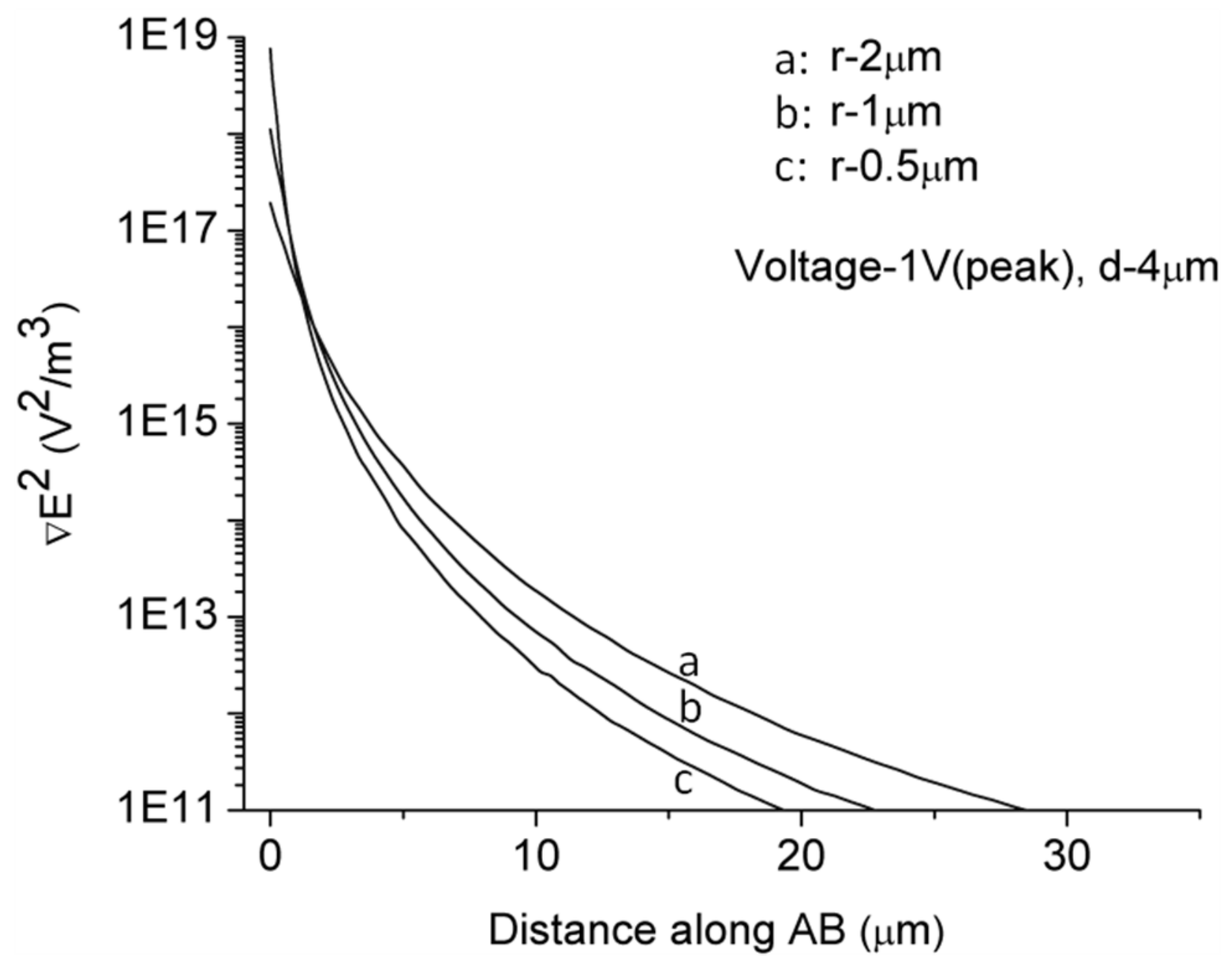


Figure 8

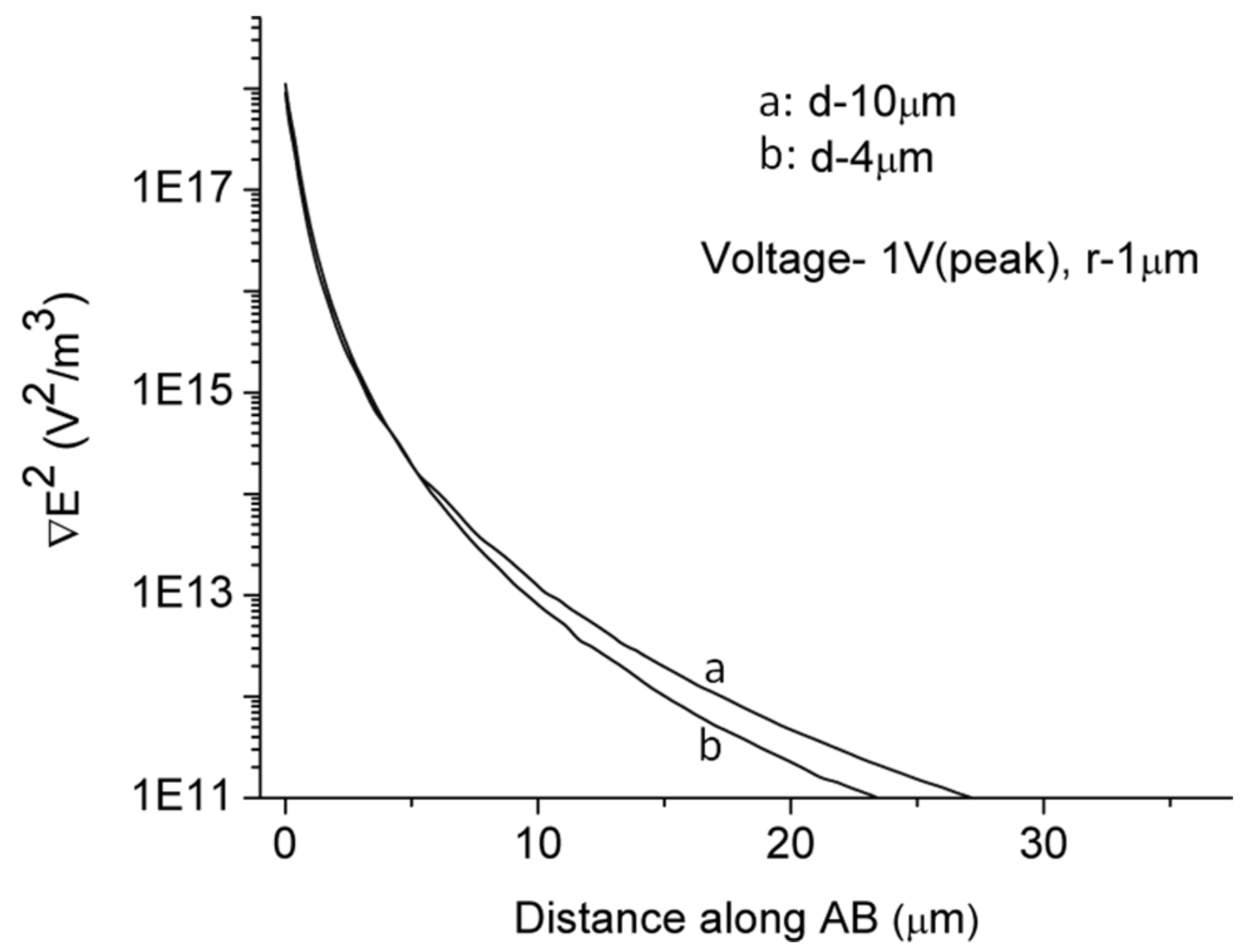

